\title{
Electron and Photon Reconstruction and Identification with the ATLAS Detector
}

\section{Emmanuel Turlay*}

LAL Orsay

E-mail: turlayelal.in2p3.fr

on behalf of the ATLAS Collaboration

The status of the reconstruction and identification of electrons and photons in the ATLAS detector is presented. Electrons and photons are crucial for both a thorough understanding of the detector and a wide variety of physics studies. An electron identification efficiency of $64 \%$ can be reached for a jet rejection of $10^{5}$. The electromagnetic energy scale can be obtained in-situ with a permil accuracy once the material in front of the electromagnetic calorimeter will have been verified with high accuracy.

The reconstruction process is described along with the identification and calibration techniques developed to obtain the required performance.

2008 Physics at LHC

September 29 - 4 October 2008

Split, Croatia

\footnotetext{
* Speaker.
} 


\section{Introduction}

Electrons and photons are of major importance for many studies at the LHC, be it for the understanding of the detector, the measurement of Standard Model processes or the search for new physics.

In order for signatures including electrons or photons to be reconstructed clearly in an environment dominated by the large jet cross-section, high identification efficiencies must be achieved along with unprecedented QCD rejections. The analysis of multi-lepton final states $(H \rightarrow 4 \ell$ or $J / \psi \rightarrow e e$ ) on one side and the search for new high $-p_{T}$ signatures on the other side require an efficient identification of electrons with transverse momenta ranging from $5 \mathrm{GeV}$ to several $\mathrm{TeV}$. Also, the energy lost by electrons undergoing bremsstrahlung in the inner detector should be recovered. For the mapping of the material before the electromagnetic calorimeter as well as the study of multi-photon final states $(H \rightarrow \gamma \gamma$ or $G \rightarrow \gamma \gamma$ ), a good reconstruction of photon conversions over the whole acceptance of the inner detector is necessary.

The physics goals for ATLAS include the rediscovery of many Standard Model candles (electroweak vector bosons, top quark, etc...). If the Higgs boson or supersymmetry exist, many observables including electrons or photons will have to be measured with excellent resolution and efficiency. For these purposes, a good directional and energy resolution as well as a precise knowledge of the electromagnetic energy scale are necessary. This can be achieved by a combination of calibration techniques involving both Monte Carlo based and in-situ methods.

The ATLAS experiment expects to see its first collision data in the summer of 2009. The performance of the reconstruction and identification as estimated from Monte Carlo will then have to be confronted to the measurements from real data [1, 2].

\section{Reconstruction}

Track reconstruction ATLAS uses two complementary algorithms to reconstruct tracks. The standard technique proceeds inside-out. It exploits the high granularity of the precision detectors to find prompt tracks originating from the interaction region. Track seeds are found in the pixel and $\mathrm{SCT}^{1}$ layers and are then extended into the $\mathrm{TRT}^{2}$. This method is very efficient for tracks left by primary charged particles. The second method called back-tracking proceeds reversely. It searches for unused track segments in the TRT and extends them into the SCT and pixel detectors when possible to improve the reconstruction efficiency for secondary tracks from conversions or decays of long-lived particles. The track reconstruction efficiency for high- $p_{T}$ electrons is close to $100 \%$ and uniform as a function of $|\eta|$ up to $|\eta| \approx 1.4$ where the material in the inner detector increases substantially. It is also fairly uniform as a function of $p_{T}$ down to low values, with $97 \%$ reconstruction efficiency for $10 \mathrm{GeV} p_{T}$ single electrons averaged over all $|\eta|$.

As the amount of material in the inner detector increases, the probability for an electron to undergo bremsstrahlung rises. The energy lost in the process can be recovered with dedicated fitting algorithms. The dynamic noise adjustment (DNA) method extrapolates track segments to the next layer

\footnotetext{
${ }^{1}$ Semi-Conductor Tracker. Part of the precision layers.

${ }^{2}$ Transition Radiation Tracker. The outtermost part of the inner detector only provides azimuthal information.
} 


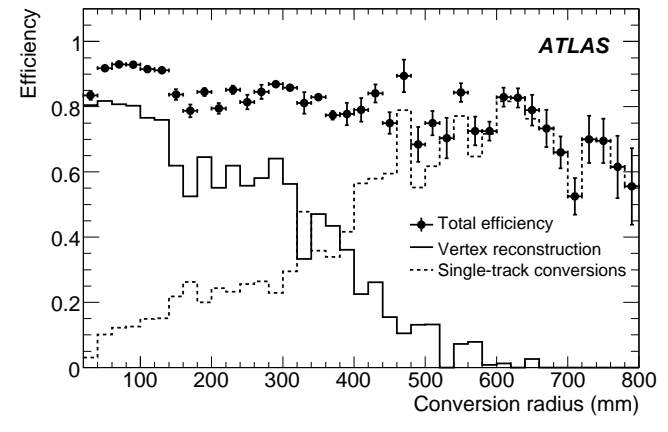

Figure 1: Conversion reconstruction efficiency versus conversion radius combining vertex and single-track reconstruction [2].

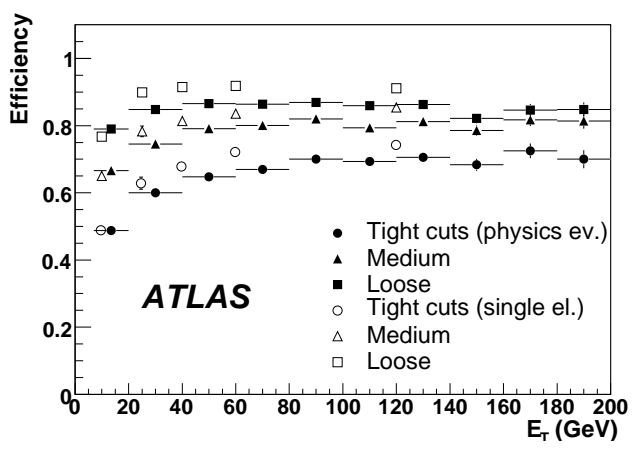

Figure 2: Electron identification efficiency versus transverse momentum within $|\eta|<2.5$ for single electrons and electrons from supersymmetric events [2].

while allowing for energy losses compatible with a hard bremsstrahlung. The Gaussian-sum filter (GSF) acts as a weighted sum of Kalman filters operating in parallel.

Conversions Reconstructing conversions is crucial for both the mapping of the material in the inner detector and the study of di-photon final states. Primary photons have a 20 to $60 \%$ probability of converting before reaching the calorimeter. Due to the structure of the ATLAS tracker, photons which convert within $300 \mathrm{~mm}$ of the beam axis may be reconstructed with a high efficiency with standard (inside-out) tracking, while photons which convert further out may only be reconstructed using outside-in tracks. As shown in figure 1, the total efficiency averaged over $|\eta|<2.5$ spans from $90 \%$ to $65 \%$ for $R=0$ to $800 \mathrm{~mm}$.

Electron/photon separation The reconstruction of medium to high- $p_{T}$ electrons and photons is seeded in the calorimeter, whereas that of soft electrons is seeded in the inner detector. For the former, seeds are defined as any transverse energy deposit in the calorimeter above $3 \mathrm{GeV}$. Then, for each cluster, a matching track is searched for. The track is required to match the cluster within a broad $\Delta \eta \times \Delta \phi$ window and the ratio $E_{\text {cluster }} / p_{\text {track }}$ should be lower than 10 . If the track does not belong to any reconstructed conversion the object is selected as an electron candidate. If there is no matching track or it belongs to a conversion the object becomes a photon candidate. Approximately $93 \%$ of true electrons with $p_{T}>20 \mathrm{GeV}$ and $|\eta|<2.5$ are selected as electron candidates. Once the electron/photon separation has been completed, clusters are calibrated according to the nature of the object. Clusters for electrons are wider in $\phi$ than those for photons to include potential energy losses due to bremsstrahlung.

\section{Identification}

Electrons and photons will be identified initially through a robust cut-based analysis and later through various multi-variate techniques such as a likelihood ratio method or a covariance matrix technique. All methods rely on common identification variables. Those based on calorimeter measurements include the leakage of the shower in the hadronic calorimeter, the shape of the shower 


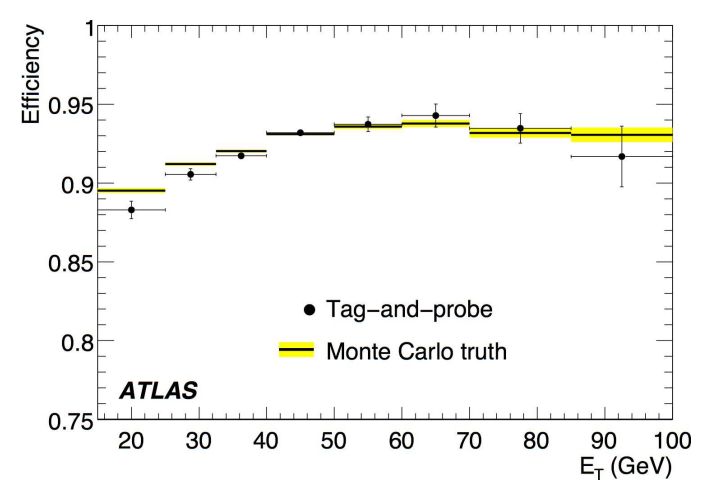

Figure 3: Electron identification efficiency as a function of $E_{T}$ as obtained from the standard Monte Carlo method in yellow and using the tag and probe method.

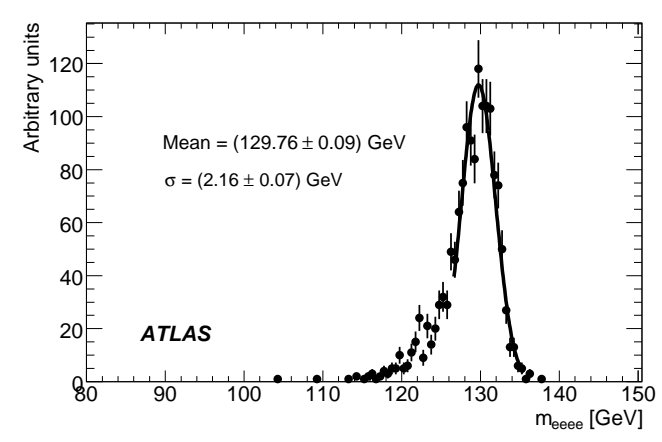

Figure 4: Invariant mass of the four electrons selected in the $H \rightarrow 4 \ell$ analysis with $m_{H}=130$ $\mathrm{GeV}$.

in the electromagnetic calorimeter and the isolation of the cluster. Those based on the track reconstruction, for electrons only, include track quality measurement and further matching constraints. For photons, an additional isolation criterion using tracks reconstructed in the inner detector is applied.

Three qualities of electrons are established in order to provide optimal signal efficiency as a function of the jet rejection, especially in the case of multi-electron final states. Loose electrons are identified using only a part of the calorimeter variables, yielding an efficiency of $88 \%$ for a jet rejection of $\sim 600$. Medium electrons use all calorimeter cuts and some tracking cuts, with an efficiency of $77 \%$ and a rejection of 2200 . Tight electrons use all available cuts for an efficiency of $64 \%$ and a rejection of $10^{5}$. The quoted efficiencies are given for electrons from $Z \rightarrow e e$ decays an have also been verified for electrons in the busy environment of supersymmetric events, as shown in figure 2. As expected, the efficiency is uniform for $p_{T}>40 \mathrm{GeV}$ and is slightly lower in supersymmetric events du to the high multiplicity of hadronic jets in the final state. Rejections are measured on filtered di-jets events. The electron efficiencies can be measured directly from data using e.g. $Z \rightarrow e e$ decays and the so-called tag-and-probe method. Electron pairs with invariant mass close to $m_{Z}$ are selected and the tag electron is required to satisfy tight cuts. Various components of the efficiency can then be measured using the second (probe) electron. Figure 3 shows good agreement between identification efficiencies obtained directly from the Monte Carlo truth information and measured using the tag-and-probe method. With $100 \mathrm{pb}^{-1}$, efficiencies are expected to be measured with a permil statistical uncertainty and a $1.5 \%$ systematic error.

Photons with $E_{T}>40 \mathrm{GeV}$ are reconstructed and identified with an efficiency of $84 \%$ for a $\sim 8,000$ jet rejection.

Identification variables can also be combined into multivariate discriminants such as a likelihood ratio or the covariance matrix method. For electrons, the use of the likelihood ratio increases the jet rejection by $60 \%$ for the same identification efficiency as the cut-based method whereas for the same rejection, the efficiency increases by $10 \%$. 


\section{Calibration}

In the ATLAS reconstruction chain, a series of corrections are applied to calibrate both the energy and position measurements. These corrections are derived from Monte-Carlo simulations and validated using test-beam data. On top of these corrections, a precise inter-calibration derived from $Z \rightarrow e e$ events will be applied.

Offline calibration starts with the correction of cell measurements to take into account electronics non-linearities and non-nominal high voltage settings. Then, once clusters are built, their energy and position are calibrated. The position of the cluster is defined as the energy-weighted barycentre calculated from the centre of the cells. Due to the finite granularity of the readout cells, the etameasurement is biased towards the centre of the cells, the so-called S-shape bias. To derive the necessary correction, the calorimeter is divided in $\eta$ into different regions based on where the behaviour of the correction displays a break in continuity. Within each region, an empirical function is constructed to describe the correction, and an unbinned fit is performed to simulated data for a particular cluster size, type and energy. The measurement of the cluster $\phi$-position must also be corrected. In contrast to the $\eta$-direction, the accordion geometry results in energy sharing between cells in the $\phi$-direction, which washes out the $\mathrm{S}$-shape bias. There remains, however, a small bias in the $\phi$-measurement which depends on the average shower depth with respect to the accordion structure. An $\eta$-dependent offset is applied.

The standard method to calibrate the energy of photons and electrons from simulated data is based on detailed Monte Carlo simulations. Dedicated simulations record the energy deposits in both active and dead materials. These quantities are then correlated to the reconstructed energy using a parametrisation. This most precise method yields a 1 to $3 \%$ energy resolution for $100 \mathrm{GeV}$ electrons versus $\eta$ and a linearity bette than $0.5 \%$ in the barrel for electrons with energy below 500 $\mathrm{GeV}$. Figure 7 shows the Higgs boson mass peak as reconstructed from the invariant mass of the four electrons in the $H \rightarrow 4 e$ channel, resulting in a $1.5 \%$ resolution and $0.7 \%$ accuracy of the peak position.

It is also possible to provide a constraint on the absolute energy scale by inter-calibrating the calorimeter using real $Z \rightarrow e e$ events. A constant term of $0.7 \%$ and a precision on the energy scale of $0.2 \%$ can be achieved by fitting the reconstructed $Z$ peak to a reference lineshape for each $\eta \times \phi$ region for $200 \mathrm{pb}^{-1}$ of data, corresponding to 160,000 recontructed $Z \rightarrow e e$ decays. Such a precision will only be achieved once the material in front of the electromagnetic calorimeter will have been determined to a high accuracy using e.g. photon conversions.

The first sources of photons and electrons are expected to arise from $\gamma$-jet processes and $b / c$ leptonic decays respectively. Also, large electron pair signals will be available from $J / \psi, \Upsilon$ and $Z$ decays with respectively $20,000,5,000$ and 3,000 events for $10 \mathrm{pb}^{-1}$. These latter signals provide clean samples to measure efficiencies in-situ and confront different reconstruction and identification algorithms.

\section{Summary}

Electrons and photons are key signatures for many physics channels, both for the assessment of the detector performances and for the search for new physics. ATLAS will reconstruct and 
identify electrons and photons with reasonably high efficiencies and very high jet rejections using a combination of calorimeter-based variables and track-based quantities (for electrons). Work currently focuses on trigger menus for early data-taking, material mapping with conversions, datadriven calibration and efficiency measurements and other performance studies which will quickly become feasable with early data.

\section{References}

[1] ATLAS Collaboration, "Expected Performance of the ATLAS Experiment, Detector, Trigger and Physics" (2008) - CERN-OPEN-2008-020

[2] G. Aad et al. [ATLAS Collaboration], "The ATLAS Experiment at the CERN Large Hadron Collider," JINST 3 (2008) S08003 